\title{
Infant responses to approaching objects: An indicator of response to distal variables*
}

\section{T. G. R. BOWER, University of Edinburgh, Scotland and}

\section{J. M. BROUGHTON and M. K. MOORE, Harvard University, Cambridge, Massachusetts}

Infants show an adaptive avoidance response to approaching objects. The response is affected by the closeness and speed of approach. It is mediated by visual variables. Air-pressure changes do not elicit the response. This kind of response implies discrimination and response to distal variables rather than merely to their proximal mediators.

Numerous investigators have used response to an approaching object as an index of ability to perceive the third dimension of space. Previous investigators have focused on one response, the blink, and one psychophysical control, dissociation of changes in visual stimulation from changes in air pressure. To close one's eyes as an object approaches is a maladaptive act. The origins of such response are of interest. However, it might be as well to look first for adaptive responses to approaching objects before investigating the origins of a maladaptive response. Similarly, it is unwise to focus on changes in visual stimulation alone if one is interested in perception of approach of an object. In the natural world, the approach of an object produces a complex of visual changes which specify approach with high ecological validity; an approaching object also produces changes in air pressure whose gradient specifies approach with an ecological validity which is probably as high as that of the visual stimuli. It is possible that the response to an approaching object can be released by either of these stimulus systems. It is also possible that the two systems cooperate synergistically as a Gestalt to release the response. Such considerations indicate that a representative design is a more appropriate research paradigm, at least while the preliminary stage of response definition is under way.

The research reported here was concerned with the development of response to approaching objects in human infants aged 6 days and up. The infants were seen during sessions of a continuing longitudinal study. They were brought into the laboratory on an ad lib schedule whenever it suited their mothers to come. All of the mothers cooperated in bringing their infants at times when they were maximally attentive. Many infants were fed in the laboratory and so were seen immediately pre- or postfeeding. The majority of the infants seen had been delivered without medication.

\section{EXPERIMENT 1}

In the first phase of the research, 21 infants aged 6 to 20 days served as Ss. The aim at this stage was simply to discover what infants do when objects approach them. A wide variety of postures, objects, and methods of moving objects were sampled. Two Os recorded behavior, aided by film records.

\section{Results}

An adaptive response to approach was discerned. In its full form, the response consisted of three components: (1) eyes open wide; (2) the head goes back; (3) both hands come up between object and face (see Fig. 1). During the response, eyes remained straight ahead. After completion of this three-part response, blinking occasionally occurred up to $7 \mathrm{sec}$ later. However, the blink seemed to be in no way integral to the adaptive response but rather part of the process of recovery from that response.

The response, as described, was affected by posture. The full form of the response occurred only when the $S$ was upright with

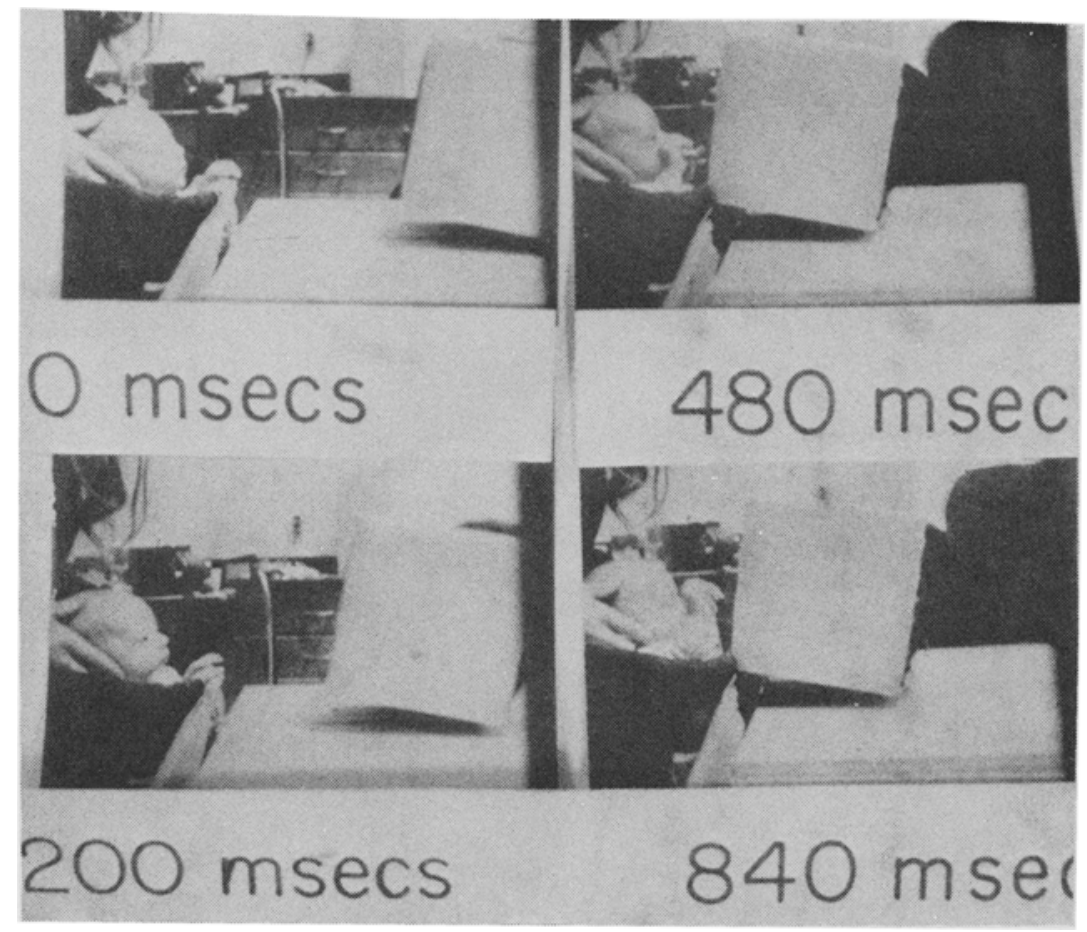

* Supported in part by an NIH grant through Harvard University and by the Medical Research Council through Edinburgh University (G 969/559/C).

Fig. 1. Photographic record of the response in a 10-day-old baby. 
his arms free and his head relatively unsupported. In order to facilitate observations, a seat was made from foam rubber pads which supported the infant up to the armpits, leaving the arms free. The seat prevented gross head movements in any direction, while also providing support for the head if it wobbled from a strictly vertical position. In postures other than upright, only abbreviated versions of the response could be seen. Simple mechanical factors would obviously rule out Component 1 -the head withdrawal-from occurring in prone or reclining postures. In such postures, the head is fully supported and simply cannot move back. It was also more difficult, though not impossible, to record Component 3 while the infant was prone or reclining, for the simple reason that at the start of any trial the infant's hands were already quite likely to be between face and object.

In addition to these factors, which would tend to obscure the response, it would seem from the work of Prechtl ${ }^{1}$ that infants in this age range never attain a fully wakeful state while lying on their backs. If this is so, the lack of vigilance associated with the posture might result in failure of registration of the approaching object.

\section{EXPERIMENT 2}

In the preliminary work, it appeared that the closer the approach, the more likely it was to elicit the response. This was studied more systematically in the following experiment.

Five infants aged 8 to 17 days served as Ss. Two foam-rubber cubes, one measuring $20 \mathrm{~cm}$ per side and the other measuring $50 \mathrm{~cm}$ per side, were used as objects. They were moved along a naturally marked wooden table with a regularly textured brick wall as background. A constant speed of $15 \mathrm{~cm} / \mathrm{sec}$ was used. The infants sat upright with their gaze line level with the center of the cube. All trials began with the object $75 \mathrm{~cm}$ away from the infant at the end of the table.

Two "nearness" values were used; in one, the object stopped $20 \mathrm{~cm}$ from the baby's face (far approach), in the other it did not stop until it was within $8 \mathrm{~cm}$ of the baby's face (near approach). The start point was $75 \mathrm{~cm}$ from the baby. The intended design of the experiment called for eight trial blocks: small object/far approach, large object/far approach, small object/near approach, large object/near approach, and the inverse order. Each trial block was to consist of seven approach-withdrawal cycles, with 7- to 10-sec pauses at start and reverse points. It was impossible to complete the intended design since the near-approach condition produced such violent upset that after one or two cycles it was necessary to abandon the experiment. Thus, no infant viewed the near approach of the large object. It would have been possible to introduce more $S$ s to complete other conditions. However, the degree of upset observed was such that we preferred to abandon the experiment.

\section{Results}

As was noted above, the experiment was severely truncated. The three conditions presented, if they had been run in full, would have allowed us to compare the effectiveness of closeness with that of retinal image size, since the small cube on its near approach subtended the same visual angle, and displaced the same amount of air, as did the large cube on its far approach, so that if image size and air movement were the effective variables we would expect these two presentations to be equally effective, whereas if closeness of approach were the effective variable we would expect the small object at the near approach to be more effective. The latter, in fact, occurred, to such an extent that the experiment could not be completed.

\section{EXPERIMENT 3}

Two further studies were made to disentangle the relative contributions of visual changes and air-movement changes. In the real world, the approach of an
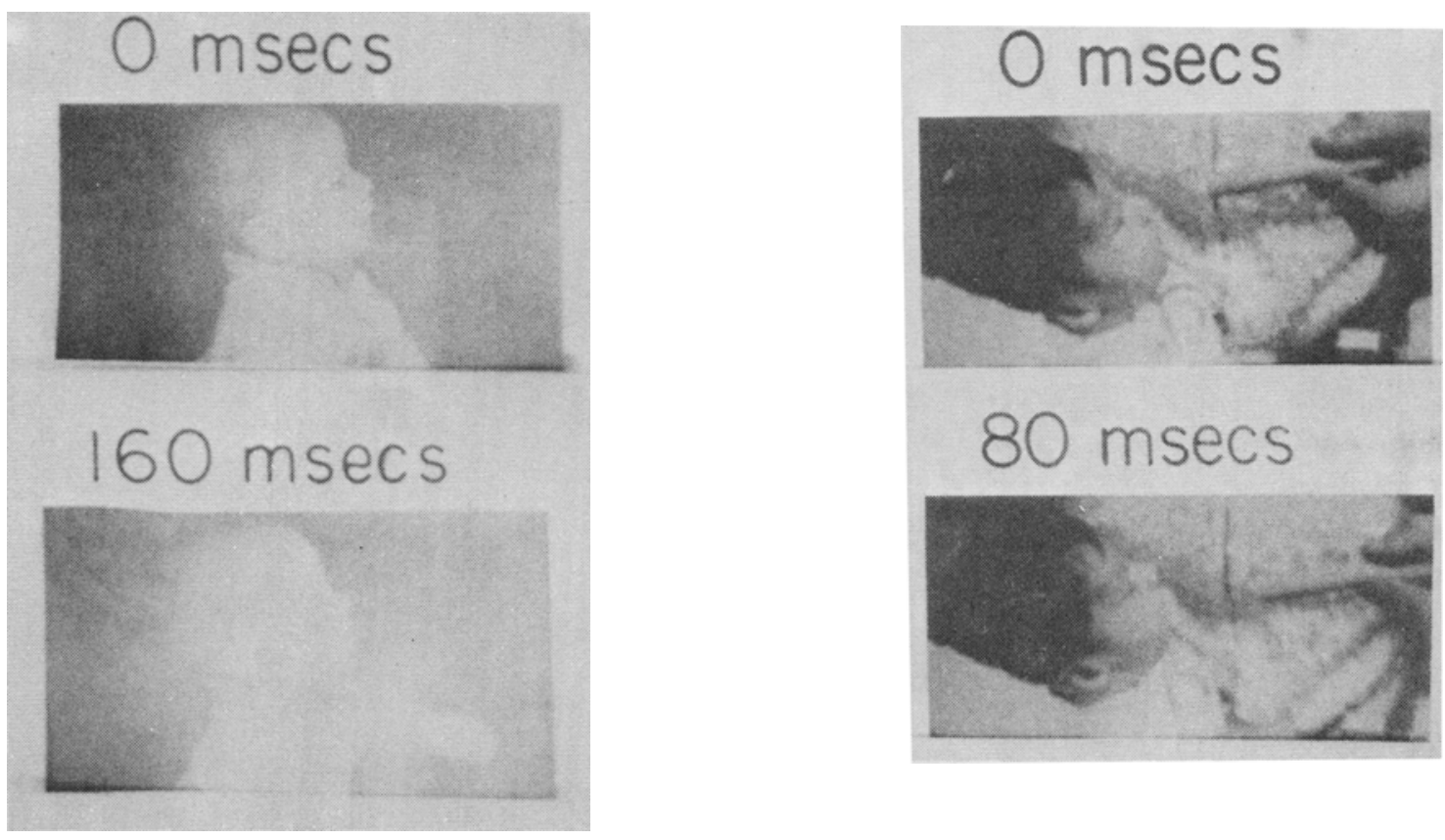

Fig. 2. Photographic record of the response to optical expansion in the same baby.
Fig. 3. Response to air-pressure gradient. 
object produces a complex of changes in stimulation, all of which specify approach of an object. Among the visual changes are optical expansion, changes in binocular parallax, and progressive foreplace occlusion. Changes in motion parallax gradients are also possible but unlikely to be effective, given the durations of movement used here. For infants of the age studied here, changes in accommodation or accommodative blur are unlikely to be relevant since the infant eye does not accommodate much and is too short and too stopped down for accommodative blur to occur or to change with distance.

Had we wished to examine visual factors as a complex, all that would have been necessary was to interpose a glass sheet between infant and approach object. However, it seemed more useful to isolate the visual factors to try to discover if any one of them was prepotent. There are theoretical reasons to believe that the optical expansion pattern should be prepotent in young human infants. Binocular parallax is affected by growth processes. Interocular separation increases with age, while the angle between the foveal axis and the optical axis of the eye decreases with age. The existence of such growth processes implies that binocular parallax must be calibrated against growth-independent variables, the optical expansion pattern and motion parallax (Bower, in press). As was said earlier, there is not enough time for motion parallax to be picked up in the approach situation. As for foreplane occlusion, it is difficult to treat it as a stimulus separate from the

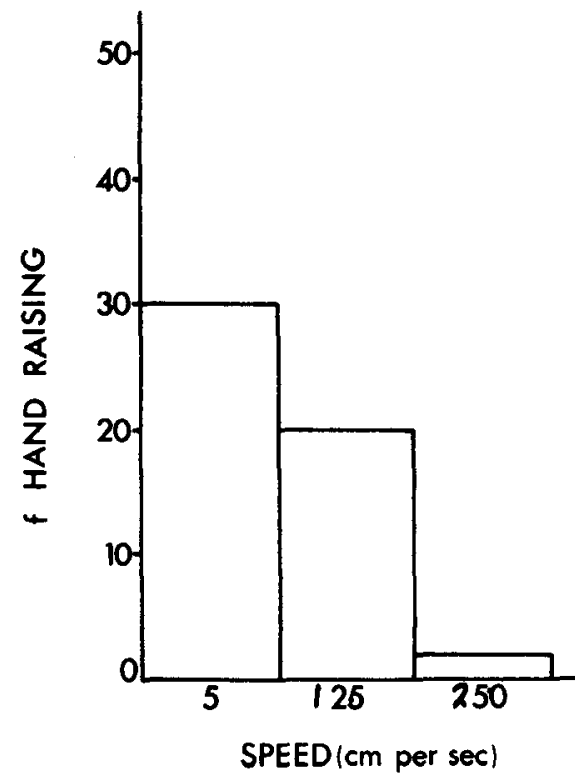

Fig. 4. Total frequency of occurrence of Component 3-hand raising-in response to optical expansion pattern alone. expansion pattern. One cannot present an optical expansion pattern which does not produce occlusion, nor can one present a foreplane occlusion without also presenting an optical expansion. For these reasons, the optical expansion pattern was chosen as the purely visual presentation.

The expansion pattern was projected onto a $6 \times 6 \mathrm{ft}$ screen. A 100-W point source was placed $26 \mathrm{~cm}$ behind the screen. The source was mounted on a clear Plexiglas table in which a grooved track was cut. The object-a red-and-black bullseye-was initially at the screen. On each trial cycle, the object moved back to the point source, stopped for $10 \mathrm{sec}$, and then moved back to the screen. The intercycle interval was $10 \mathrm{sec}$. Three speeds were used: $5,12.5$, and $25 \mathrm{~cm} / \mathrm{sec}$; four cycles of each speed were presented. Order of presentation of speeds was counterbalanced across infants. The infants sat upright, conjugate with the point source. Viewing was binocular, so that both eyes received the same expansion pattern with no binocular parallax difference. In the real world, there would be binocular parallax differences associated with the optical expansion.

Nine infants aged 10 to 20 days served as Ss. All except one were naive to approach situations, although not to the laboratory.

\section{Results}

Eight of the infants showed avoidance responses. No full-scale avoidance responses occurred to the reduced visual presentation, although the same components were discernible (see Fig. 2). The intensity of the effect was less in the purely visual situation. No $S$ was reduced to tears by the purely visual presentation. The response declined over trials. This never happened with the real object approach.

\section{EXPERIMENT 4}

The lower intensity of response could have been due to the absence of air-pressure changes. Accordingly, we presented air-pressure changes alone. Four infants served as Ss. The infants sat upright facing an air hose. The air pressure was switched on and stepped up, and then switched off. It was impossible to duplicate exactly the gradient of air-pressure change produced by the real object movement. A series of seven approximations was used.

The air movement alone produced a response (see Fig. 3) quite opposite to that produced by an approaching real or optical object. It consisted of partial eye closure, ducking forward of the head, with occasional side-to-side turning. This response was present in all of the infants seen.

\section{DISCUSSION}

These experiments show that neonate infants display a functional distally appropriate avoidance response to approaching objects. They also show that visual stimuli control the response. These results help to reconcile the differences among pediatricians, such as Sheridan ${ }^{2}$, who have found that response to an approaching object is a useful indicator of visual function and psychologists, such as White (1963), who have asserted the opposite. White focused on the blink, which seems, at least in the age range studied here, to be an associated rather than an integral component of the response. He also studied supine infants who were thus mechanically prevented from showing the avoidance response studied here. White also used an extremely high approach speed. The second experiment described here indicated that the response was already dropping off at a presentation speed of $25 \mathrm{~cm} / \mathrm{sec}$ (Fig. 4), much slower than that used by White. This probably reflects the limited data rate of the infant visual system (Bower, 1966; Trevarthen ${ }^{3}$ ). The results presented here thus indicate that, under appropriate conditions of stimulation, response to an approaching object can be a useful indicator of visual function.

These results are also of theoretical interest. Studies abound showing that infants can discriminate spatial variables. None of these studies has anything to say about whether infants can discriminate distal variables or whether they are merely responding to proximal variables per se. Thus, it has been shown that infants will preferentially fixate solids over two-dimensional representations (Fantz, 1961). The discrimination is mediated proximally by such variables as motion parallax and binocular parallax. The preferential fixation data cannot tell us whether the infants are fixating solids, as mediated by motion parallax and binocular parallax, or motion parallax and binocular parallax per se. Likewise, operant measures have been used to infer that infants can discriminate distance on the basis of motion parallax (Bower, 1966). The same results could be used to show that infants discriminate motion parallax itself, with no distal reference. Discrimination measures such as these can never answer the question of whether the organism is responding to distal or to purely proximal variables. That question can be answered only if one can discover distally appropriate responses coordinated with distal variables. Thus, when Lashley discovered that rats will grade their force of jump to distance to be jumped, everyone accepted that the rats were responding to the distal variable, 
distance, however mediated. Likewise, when Gibson and Walk (1960) showed that infants of 6 months or so will not crawl over the deep side of a visual cliff, it seemed obvious that they were responding to depth, however mediated. In all of these cases, the response is functional or distally appropriate to the distal stimulus. In the present experiments, one can assert, in the same way, that the observed response is distally appropriate to the stimulus, that it is response to the approach of an object, as mediated by such visual variables as the optical expansion pattern, but is not merely a response to these variables.

\section{REFERENCES}

BOWER, T. G. R. The visual world of infants. Scientific American, 1966, 215, 80-92.

BOWER, T. G. R. Morphogenetic factors in space perception. In D. Hamburg and K. Pribram (Eds.), Proceedings of the Association for Research in Nervous and Mental Disease 1969. in press.

FANTZ, R. L. The origin of form perception. Scientific American, 1961, 204, 66-72.

GIBSON, E. J., \& WALK, R. D. The visual cliff. Scientific American, 1960, 202, 64-71.
WHITE, B. L. Development of perception during the first six months. Paper read at the American Association for the Advancement of Science, December 1963.

\section{NOTES}

1. Personal communication, 1970.

2. Sheridan, M. Personal communication, 1968.

3. Trevarthen, C. Unpublished manuscript, Center for Cognitive Studies, Harvard University, 1968.

(Accepted for publication October 28, 1970.) 